\title{
Mechanisms, resources, and background conditions
}

\section{Colin Klein ${ }^{1}$ (1)}

Received: 30 September 2017 / Accepted: 25 September 2018 / Published online: 28 September 2018 (c) Springer Nature B.V. 2018

\begin{abstract}
Distinguishing mechanistic components from mere causally relevant background conditions remains a difficulty for mechanistic accounts of explanation. By distinguishing resources from mechanical parts, I argue that we can more effectively draw this boundary. Further, the distinction makes obvious that there are distinctive resource explanations which are not captured by a traditional part-based mechanistic account. While this suggests a straightforward extension of the mechanistic model, I argue that incorporating resources and resource explanations requires moving beyond the purely local account of levels that some mechanists advocate.
\end{abstract}

Keywords Mechanism · Resources $\cdot$ Background conditions $\cdot$ Explanation $\cdot$ Levels

\section{Parts and resources}

Back in the day, my car began to overheat. Potential explanations fell into two categories. First, a mechanical failure: a stuck thermostat, a failing pump, a blocked radiator. Second, a problem with the coolant itself: contamination, age, leaks. Any of these, if true, would be part of a perfectly respectable mechanistic explanation of my car's overheating. Yet qua explanations, they also have importantly different properties.

The first set of explanations appeals to what I will call mechanical parts: discrete entities with well-defined roles to play. Mechanical parts are individually present or absent. They work or they don't. The second set of explanations, by contrast, appeals to a resource that is used by mechanical parts in the course of performing their roles. Resources usually have a quantity and a quality: we ask how much coolant the car has, and what degree of contamination it might show. Resources often play multiple roles. The very same stuff is involved in cooling the engine and heating the passenger compartment.

Colin Klein

colin.klein@anu.edu.au

1 School of Philosophy, Australian National University, Canberra, ACT 0200, Australia 
This paper has two goals. First, it will clarify and argue for the distinction between mechanical parts and resources, and show the important role of the latter in mechanistic explanations. Second, it will show that resources have some features that make them look more like background conditions. By separating them off and detailing their role, we thus find it easier to draw the distinction between background conditions and true mechanical components (which include both mechanical parts and resources). Hence by showing how resource have distinct features-while still remaining under the broadly mechanist umbrella-we make the problem of carving off background conditions much easier.

To motivate this position, I begin with a persistent problem for the mechanist: that of distinguishing mechanical components from background conditions.

Suppose we explain how a car engine works. That explanation will assume (usually tacitly) that the engine is properly lubricated, in an environment with an ambient temperature in a certain range, and so on: these are mere background conditions that are necessary for the mechanism to work, but which are typically contrasted with the mechanical components that actually do the explaining. One would like that distinction to be principled, and so a good account of mechanistic explanation requires a criterion to distinguish background conditions from mechanistic components.

Distinguishing background conditions runs into two distinct problems that ought to be distinguished. First, mechanistic explanations are a species of causal explanations, and all causal explanations must distinguish background conditions from the meatier explanans. This is a well-known problem for any causal theory of explanation, one that has attracted a lot of attention (Menzies 2007). For that reason, I set it aside. I am interested in a second problem that is specific to the mechanistic project.

Mechanistic explanations, at least in contemporary formulations, appeal to local decompositions of target phenomena (Craver 2007; Bechtel 2008). Explaining the activity of a target object involves appeal to the activity of some of its spatiotemporal parts. Not all parts are equally relevant, though. If a wombat crawls into my engine compartment and goes to sleep, that doesn't make it a mechanistic component of my engine-even if it has a variety of causal interactions with the engine. The criterion that rules out the wombat thus needs to rule out spatiotemporal parts that aren't parts of the mechanism, even if they are causally related to the mechanism. That needs to include mere background conditions as well.

This is arguably a distinct, and harder, problem than the one that all causal theories face. The standard problem of background conditions starts with a well-delineated set of potential causes, and asks which we should prefer. The mechanist, however, must also find a way to delineate the potential causes in the first place. That is the second, and more pressing, problem of background conditions.

I focus on the account of mechanistic explanation given by Carl Craver. A mechanism, in Craver's $(2007,5)$ formulation, is "a set of entities and activities organized such that they exhibit the phenomenon to be explained." Mechanistic explanations are fundamentally multi-level. They work by demonstrating that the behavior of a whole can be captured in terms of the organized activities of its parts $(2007,139)$.

Craver offers the relationship of constitutive relevance in order to distinguish the true mechanistic components. Constitutive relevance is in turn cashed out in terms of mutual manipulability. Some proper part $x$ of $S$ is constitutively relevant to $S$ 's 
$\psi$-ing if there is an intervention on $x$ 's $\phi$-ing that would change $S$ 's $\psi$-ing, and if there is an intervention on $S$ 's $\psi$-ing that would change $x$ 's $\phi$-ing (Craver 2007, 153). In other words, it must be that one is able to reliably manipulate the behavior of a constitutively relevant component to manipulate the behavior of the whole, and vice-versa. The behavior of the fuel injectors can be manipulated by speeding up the engine, and the engine can be manipulated by changing the speed of the injectors; that is why the injectors count as constitutively relevant. By contrast, there's no (reliable) change I can make in the interloping wombat by manipulating the engine, and poking the wombat has an inconsistent effect on the engine.

Craver also argues that the relationship of mutual manipulability fails for mere background conditions (Craver 2007, 153). Lubricants are part of the causal chain between pushing the gas pedal and making the car go. But the manipulability relation is asymmetric and non-specific. Pressing the pedal makes no difference to the presence of lubrication. Changing whether there's sufficient lubrication makes a difference to the target phenomenon, but only by making everything break down.

Yet problematic cases remain. Consider: Forming long-term memories via longterm potentiation uses glucose, which is largely provided by astrocytic glycogen. Depleting glycogen experimentally leads to amnesia. The elderly often have memory problems, and also tend to have lower levels of glucose. Supplementing them with glucose improves their memory (Messier 2004; Newman et al. 2011). Hence forming memories depletes astrocytic glycogen, and depriving astrocytes of glycogen causes amnesia.

Hence astrocytic glycogen passes Craver's test for mutual manipulability. This seems like a problem. Intuitively, glycogen doesn't quite seem like part of the mechanism of memory formation: it's the fuel that drives the mechanism. Indeed, that's exactly what I'll claim. There is an important sense in which astrocytic glucose isn't like lubrication, ambient temperature, and so on. Instead, then, I claim that mechanical components are more diverse than they originally seemed. I argue we ought to distinguish at least two types of component: mechanical parts and resources. Resources have features that make them look like background conditions. That generates the persistent confusion. Yet they are ultimately distinguishable, and more akin to the more familiar bits cited in mechanistic explanations. This tripartite division thus does real philosophical work. To get there, however, the distinction must be sharpened.

\section{Two categories of mechanistic component}

\section{Mechanical parts}

Fuel injectors, hearts, sodium channels, and syntactic modules appear to have, on the face of it, little in common. Abstracting away a bit, though, we can see that they share several important features. Call components with these features mechanical parts.

First, mechanical parts tend to persist over the timescale of the behavior of the whole that we wish to explain. The different engine components are present the 
entire time the engine is running. That is an important feature of the mechanistic explanation: we implicitly assume that when the fuel rail sends gas to the injectors, the injectors are always there and ready to receive it. Of course, on longer timescales a part may break down; the important thing is stability over the course of the phenomenon to be explained.

Conversely, the failure of a mechanical part to persist over an appropriate timeframe is often the explanation for the failure of the whole mechanism to perform its job. The Challenger exploded rather than went into space because an O-ring failed. Tetrodotoxin kills by blocking the activity of sodium channels. Mechanical parts must thus be available and functional throughout the timescale of the explanation. Failure to do so explains deviations from the norm.

Second, mechanical parts are individually important. In addition to the availability of component-types, the identity of a component over time is often crucial to the explanation. My car starts hard in the morning. Why? It is too old to take ethanolaugmented gasoline. The ethanol destroyed the check valve in the fuel rail. As it sits overnight it loses pressure and makes for a hard start. Note that for this explanation to work, it is important that the same entity was affected by the ethanol, leaked overnight, and therefore explains what happens the next day.

Thus mechanical parts don't just persist, but can persist as the very same entity over a series of activities. This is often obscured when we give mechanistic explanations because we often care about explaining types of mechanisms rather than individual tokens. But for any individual token mechanism, it is usually assumed that the same components persist, and persist as the same components, over time.

Third, mechanical parts are typically portrayed as causally conservative. That is, each mechanical part interacts with only a limited subset of the other components in a small number of ways. The fuel rail does one thing, the intake another, the injectors a third; further, the fuel rail interacts only with the fuel line and the injectors, the injectors with the rail and the chamber, and so on. Causal conservativeness allows for modularity, which is a feature of complex mechanisms (Simon 1996; Bechtel and Richardson 2010). Further, mechanistic decomposition is itself aided by the causal conservativeness of the individual components. Most of the processes we care about are complicated. We explain a complicated process by looking at the components involved and the limited sets of relationships between them. Note that the components need not be simpler than the whole. Rube Goldberg machines show that it's possible to build up a simple process from complex components. What's important, rather, is that each mechanical part affects something less than the whole, and so we can partition out the effects of mechanical parts more easily.

Fourth and finally, our mechanistic explanations are often indifferent to the composition of the mechanical parts. Many mechanistic explanations care only about the location and characteristic activities of the mechanical parts. Mechanistic explanations often involve functional decompositions (Cummins 1975, 1983), and functional decomposition cares only about the activities of the components and their relation to one another.

Note that this is a weaker claim than saying that mechanical parts are multiply realizable, at least in the classic sense. Mechanical parts might well be constrained, and rather severely, by the environment in which they must work (Shapiro 2005). A 
catalytic converter is a functional entity, but the constraints placed by the high operating temperatures and the laws of chemistry mean that only a few expensive elements will do. The claim is rather that for many mechanistic explanations, we don't care about how the components are composed, so long as they do the job: the fuel rail is just something that manages to get fuel from the pump to the injectors. The details aren't that important, at least when we're explaining hard starts.

\section{Resources}

Not all explanations focus on mechanical parts. My engine stops running. Why? Because the car ran out of gasoline. Without gasoline, the engine doesn't go. That seems, intuitively, like a mechanistic explanation-certainly, it's on a par with the explanation of my engine's failure where I cited a bad check valve. (Most troubleshooting procedures for engine failure begin with checking the fuel gauge.) Furthermore, gasoline seems to pass the constitutive relevance condition. Some changes to how much gas there is in the car will change how the engine runs, and running the engine changes how much gas there is.

Yet gasoline is very much unlike the rest of the engine. Gasoline is transformed by the action of the engine. The engine persists through those transformations. Gasoline thus gets used by engines, while engines do the using.

Gasoline is one example of a resource. There are many resources that show up in explanations, across a variety of fields. The grass in the paddocks and water in the streams are resources for the livestock. The catalytic enzyme argininosuccinate synthase is the rate-limiting step in the synthesis of arginine. Some resources are more abstract. City planners must consider tax base, water supplies, and volume of low-cost rental units. Each are resources that a city needs to run efficiently, but each is spread over time and space. Computational complexity affects the amount of memory and processing time that an algorithm needs to run, and is affected by the number of processors available to implement the algorithm. The performance of an internet connection depends on resources like bandwidth and downstream cache. Cognitive scientists appeal to a variety of resources like working memory capacity, attention, and willpower.

Each of these in turn appear in resource explanations. We explain characteristic patterns of psychological performance and deficit by showing how psychological resources can be blocked, competed for, and otherwise made unavailable. Insufficient housing leads to negative health outcomes. Having too many browser tabs open at once uses up memory and slows down the rest of the computer.

Examples are easily multiplied. I have already presented a diverse bunch. What do they have in common? I will suggest a cluster of features, each of which complements those of mechanical parts.

First, tokens of resources need not persist across the timespan of explanations in which they feature. Cows eat grass. Engines burn gasoline. These activities irreversibly transform token chunks of resource, and that transformation is necessary for the proper functioning of the mechanism. Indeed, such transformations are often the whole point of the mechanism in question. 
More broadly, resources can be made available or unavailable for transformation. The availability of resources is often a key feature in explanations. Working memory has a certain capacity, and holding items in working memory diminishes that capacity. Streaming a movie makes it harder to download system updates, because each takes a certain amount of bandwidth.

Second, and along the same lines, token chunks of resources are not individually important. That is, it doesn't (typically) matter which bit of resource gets used, so much as there's enough of it to go around. The car factory doesn't care which ingot of steel it uses. Similarly, it does not matter (for most applications) whether you use the first sector on the hard drive or the ten thousandth.

Mechanical parts are individually important precisely because the tokens do such different things. Resources, by contrast, admit of equipotent divisions. Every bit of gas is basically as good as every other. These divisions might be effectively continuous (as with water supplies), or they might come naturally chunked (as with working memory). Each mechanistic part of the computer, by contrast, does something individually important-you can't interchange the hard drive for the memory. This distinction is roughly reflected in a linguistic division. Resources are typically picked out using mass nouns. In order to talk about some collection of them, we need to discretize them using a measure word that specifies the relevant divisions (three cups of water, twelve kilobytes of memory). Mechanical parts, by contrast, are usually referred to by count nouns, and so can take plurals without further divisions specified.

Third, most resources are causally promiscuous. (I suspect that all are potentially so.) That is, resources can interact with many different components of a complex system, and many different components have an interest in using that resource. All of the grass in the paddock is available to any of the cows that want it. Even relatively restricted resources-like, say, modality-specific cognitive buffers - are available to any process that might use that resource type.

Note that this doesn't mean that any mechanical part can causally interact with a resource, or even any part that could. Indeed, causal promiscuity is important because many resource explanations involve competition for a limited amount of resource. Conversely, many complex mechanisms have portions devoted to controlling access to resources. Multitasking operating systems spend a great deal of time mediating conflicts over access to memory and processor time. Even when resources aren't actually promiscuous, it is often because of deliberate strategies taken by mechanisms to control access, rather than the intrinsic properties of the resources themselves.

Fourth, we typically do care about the realization of resources, and otherwise similar resources can't be substituted willy-nilly. Car engines need gasoline; it would be nice if something else worked, but nothing works quite as well. Even small deviations can be problematic-recall the issues caused by ethanol-augmented gasoline. Food chains are vulnerable to collapse precisely because organisms need specific resources. Ion channels might work in a variety of ways, but it's important that they let in only one specific sort of ion. Unlike mechanical parts, then, we often $d o$ care how a resource is implemented. 
Even substitutions that maintain functional similarity in a broad sense can cause a variety of problems. Ethanol-augmented gasoline in your old car, or a substandard batch of steel in your factory, may not shut things down-but that substitution is what you'll have to cite when you want to explain why things break, fail, and otherwise fall short of expectations.

\section{Taxonomizing components}

The features I have emphasized are meant to be diagnostic rather than definitive. They represent features of mechanical parts and resources that tend to cluster together in ways that make the categories worth tracking. A particular resource or mechanical part may lack some of these features; further, as I'll argue in "Relative boundaries and the point of mechanisms" section, there is an important sense in which actual classifications themselves are crucially explanation-relative.

Nevertheless, I suggest that making this distinction is a natural extension of the mechanistic project. Consider: why appeal to mechanisms rather than simply causes? One answer, I assume, is that some sets of causes tend to persist across different types of complex objects in virtue of their parts, and to do so systematically enough to be worth tracking. Why do I pick the wire including the insulation, when it is only the copper core that carries the electricity relevant to the explanation? Presumably because insulated wires are common features of circuits, and importantly so. In general, there are many functionally equivalent spatiotemporally carvings of objects (Haugeland 1998), and we don't necessarily pick the minimal one. Hence the goal of mechanistic explanation is to taxonomize causes, clustering them into groups with similar features.

The distinction into mechanical parts and resources is a natural extension of this strategy. They are distinguished by a variety of more fine-grained features that tend to cluster together. Perhaps the broadest, most abstract taxonomic principle in the background is that between the agent and the patient of action. Mechanical parts are spoken of as agents: they do things. They might be caused to do things, but they are primarily active. Resources, by contrast, are largely patients. They are acted upon. Their passivity is why we need mechanisms to control their availability and influence (money doesn't do anything without people to move it around, banks to keep it safe, and so on).

It is possible that, for very simple mechanistic explanations, the distinctions might blur and become less useful. The ballast of a ship is an important part of the explanation of why it can stay upright in rough seas. ${ }^{1}$ On the one hand, this seems functional: ballast mostly persists, is defined in terms of its functional role, and is relatively realization-independent. On the other hand, ballast can be dumped or taken on to affect trim, and mostly just sits there being heavy. Is it a resource or a mechanical part? I suspect that there's no obvious answer. But that is because nothing very important turns on the question: the explanation itself is one where

\footnotetext{
1 Thanks to an anonymous reviewer for suggesting the example.
} 
the mechanistic style of explanation adds very little beyond enumerating the basic causal facts. ${ }^{2}$ One we get to more complex mechanisms, however, we see the differentiation into mechanical part and resource, and keeping track of the two becomes relevant again.

\section{Resource explanations}

Resources appear to be the primary explanans in a variety of mechanistic explanations. That is, there are explanations that account for the activity of the whole in more or less the same way that canonical mechanistic explanations do, but which appeal to resources rather than (or in addition to) mechanical parts.

Most obviously, many explanations must cite resources if they're to be complete. You don't know how a car factory works unless you know about the materials it uses and the machines that do the using. An explanation of how a heart works is obviously incomplete without mentioning the blood that gets pumped around. Resources thus seem to play an ineliminable causal role.

Many explanations also give resources pride of place. Some of these invoke various ways in which resources can be depleted, blocked, competed for, or otherwise made unavailable. Availability of resources thus shapes the behavior of the whole. Sometimes this relationship is crude: no gas, no driving. But the relationship can be more complex. Computational complexity theory posits specific relationships between an algorithm's properties and the amount of time, space, and so forth it can use. Many low-level assembly language algorithms are tuned to avoid accessing RAM, which can be orders of magnitudes slower than accessing in-CPU cache (Duntemann 2011). The performance of instances of the algorithms can be complex, but largely explained by the differences in access time between two distinct types of memory resource. Along the same lines, some enzymes are the rate-limiting step in reactions, and so the norm of reaction within the mechanism is largely explained by reference to the bulk availability of that enzyme.

Appeal to resources can also be evidentially important, because they serve to invalidate (or at least cast suspicion upon) certain types of mechanistic

\footnotetext{
${ }^{2}$ Indeed, for disciplines without a clear agent-patient distinction, the two carvings might not get much purchase anywhere. Simple physical explanations are an obvious example: in the world of atoms and void, things simply happen, without actor or acted upon. This may be why the mechanistic style of explanation seems less useful in the case of basic physics. This is not something special about physics per se though: more complex physical explanations might reproduce the agent-patient structure. Consider explanations of supernovas. The star uses its fuel, and once it is forced to rely on inferior resources for stellar combustion it goes nova. Having reconstructed the agent-patient structure, it is also natural to talk about the mechanisms of stellar evolution. Conversely, there are systems that resist an easy agent-patient dichotomy because they appear to have features of both at once. The most obvious of these are what Bechtel calls active mechanisms (2008, Ch8). These include the mechanisms involved in autocatalytic loops, homeostatic processes, and other feedback cycles. Key to mechanisms with feedback is the possibility of a mechanism altering its own operation-that is, acting at the same time as agent and patient. Active mechanisms are, as Bechtel argues, key to understanding many living processes; they're also arguably more important for understanding many neural processes (Bechtel 2008).
} 
explanation. Tim Shallice (1988) relies on considerations of resources in his argument that single dissociations offer only weak evidence for the distinctness of cognitive processes. Suppose I have a lesioned patient who can spell regularlyspelled English words and regular nonwords, but not irregular words. I might conclude that irregular words are handled by a different process. But as Shallice points out, there is another possible explanation: spelling irregular words might just be harder. That is, spelling might have only one route, but that route might require a general resource that is stressed more by harder tasks than by easier ones-intelligence or working memory or attention, say. Partial damage to this resource will only affect more difficult tasks. Ruling out these resource artifacts is the primary reason why double dissociations are so prized in neuropsychology, and why the combination of lexical and phonological agraphia is necessary to establish a dual-route model of spelling (Caramazza 1986; Shallice 1988).

Further, many of the techniques for distinguishing and exploring resources involve the same methods that picking out mechanical parts do. Just as it is not always obvious which spatiotemporal parts count as mechanical parts, it is also not obvious which resources a mechanism actually uses. The very same techniques for exploring mutual manipulability that Craver details in his (2007) can be applied to resources. So for example, to determine whether something is a resource you can manipulate it, block access to it, change the whole to measure the amount of the resource, and so on. Scientists do very similar things to investigate both mechanical parts and resources. The two thus deserve explanatory parity.

Finally, differences between resources can often explain differences between mechanisms. Sometimes these involve different resources as inputs. Different steelmaking processes are differentiated by what resources they use (e.g. forced air in the Bessemer process vs pure oxygen in the contemporary basic oxygen process). Differences in resources often require different mechanisms: diesel engines don't have spark plugs and have heavier combustion chambers, because diesel must be detonated rather than merely ignited. Explanations of why the two types of mechanism differ thus appeals to the different resources those mechanisms are meant to use, even if they are functionally similar at the level of the whole.

At a more abstract level, resources themselves can vary in a variety of ways. As an incomplete taxonomy: resources can be limited or unlimited, they can admit of discrete or continuous divisions, they can be permanently transformed or merely blocked, usable in parallel or in serial, and so on. These distinctions make a difference to the systems that use them. Computers with random-access memory have different properties to those with older serial-access memory. Omnivores have a different ecological profile than carnivores. Breakbulk shipping has a different timescale and efficiency curve to container shipping. The differences between these complex mechanisms is explained by the differences in the resources they use. Resource explanations are thus important when we compare mechanisms as well as when we try to account for the behavior of single mechanisms.

In sum, resources constitute their own class of explanans, distinct from mechanical parts, and sometimes form the primary or even the sole explanatory variable for the behavior of mechanisms. They are thus worthy of focus in their own right. 
Table 1 Contrasting components and resources

\begin{tabular}{|c|c|c|c|}
\hline & $\begin{array}{c}\text { Mechanical } \\
\text { Parts }\end{array}$ & Resources & $\begin{array}{c}\text { Background } \\
\text { Conditions }\end{array}$ \\
\hline Causally... & Conservative & Promiscuous & Promiscuous \\
\hline Realization & Irrelevant & Relevant & Relevant \\
\hline Individual? & Yes & No & No \\
\hline Actual Variation? & Yes & Yes & No \\
\hline Specfic? & Yes & Yes & No \\
\hline Systematic? & Yes & Yes & No \\
\hline Mutual Manipulability? & Yes & Yes & No \\
\hline
\end{tabular}

\section{Mechanistic components and background conditions}

The preceding sections focused on the differences between mechanical parts and resources. They also share a number of features, enough that both can function in mechanistic explanations. Yet there are also some important differences between the two. I suggest that the differences are shared between resources and mere background conditions. Overlooking resources as a distinct category has been one of the reasons why it has been difficult to give a precise delineation between mechanistic components and background conditions. I summarize the features of each of the three categories in Table 1.

The entries in Table 1 ought to be seen as claims about the typical or usual features of the respective kinds. The line down the center of the table indicates where resources are grouped with either mechanical parts or background conditions.

On the one hand, there are several respects in which resources are similar to background conditions. Both tend to be causally promiscuous: engine oil lubricates and cools a wide variety of components. The sun rises on the just and the unjust alike. Both facilitate mechanisms in realization-specific ways: oxygen is a background condition necessary for the struck match to light, and pretty much only oxygen will do. And both tend to be important in bulk but not individually.

Yet there are a variety of ways in which resources are more like mechanical parts. For one, both mechanical parts and resources can vary in their actual properties and activities over the timescale of explanations. This makes them, in Kenneth Waters' (2007) term, actual difference-makers. By contrast, background conditions enable a process by remaining constant over the timescale of the explanation. Similarly, as Woodward (2010) notes, causes might have more or less specific relationships to their effects. When $X$ is a relatively non-specific cause of $Y$, then "we are more likely to regard $\mathrm{X}$ as a mere enabling (or background) condition for Y" (Woodward 2010, 317).

Conversely, Campbell $(2007,2010)$ notes that explanatorily interesting variables let one make well-defined changes to a target phenomena, a relationship that I call systematicity (2017) . Background conditions don't seem to have systematic effects either. The classic background condition makes everything break down in unsystematic and uninteresting ways when it fails.

Finally, true background conditions don't typically pass the mutual manipulability test. I might be able to affect my engine's functioning by changing the local gravitational field, but I can't change gravity by running the engine (Craver 2007). By contrast, resources and mechanisms do pass the mutual manipulability test. 
Ultimately, while resources have much in common with background conditions, it is the cluster of properties they have in common with mechanical parts that are most relevant for explanation.

With that in mind, return to the problem case with which I began. Recall that astrocytic glycogen is a crucial energy source for long-term memory formation. Forming memories depletes astrocytic glycogen, and depriving astrocytes of glycogen causes amnesia. So astrocytic glycogen satisfies Craver's mutual manipulability criterion. Further, there is a specific and systematic relationship between glycogen and performance on memory tasks (Messier 2004; Newman et al. 2011): a little depletion causes a little deficit, more causes more, and so on. So astrocytic glycogen passes these tests as well. Finally, astrocytic glycogen actually varies in populations we care about, and that actual variation is part of the explanation of actual differences between mechanisms in that population. Crucially, the relationship between glucose and LTP is not a simple on-off sort of relationship: the relationship is a specific one, in the sense specified by Woodward (2010). That is, there are many distinct possible levels of glucose, and a (roughly) one-one mapping between those levels and the efficiency of LTP within a certain range.

Of course, like most resources, glycogen is available to many processes (and sub-processes within memory formation itself), is realization-specific, and is important only in bulk. That explains why it seems like a mere background condition, and why the line was difficult to draw. Indeed, I suspect that some of the difficulty in coming up with an adequate characterization of background conditions (at least for mechanistic explanations) stems from the fact that mere background conditions often resemble resources. All the more reason, then, for philosophers to distinguish the resources.

\section{Relative boundaries and the point of mechanisms}

I conclude with a reflection on the ontological status of mechanistic components. I've spoken of objects as if they are either mechanical parts or else resources, and that this categorization can be made in some straightforward, objective sense. The world is messier than this. The distinction between part and resource is relative to our explanatory interests. The butcher doesn't care which kidney is which, and sells them by the pound. The anatomist makes finer distinctions, cares more about functional connections, and studies kidneys in place and individually.

Does this undermine the distinction? I think not. So far, I have considered the differences between resources and mechanisms within the context of explaining a single phenomenon. It is a standard part of the mechanistic project that fixing the explanandum is an important step in fixing the decomposition of a mechanism into components. The same applies to the categorization of components as mechanical parts or as resources: having settled on a phenomenon we want to explain, parts within the causal structure that gives rise to the phenomenon can be categorized as one or the other. Hence relativity is not problematic so long as it is well-behaved. That is, so long as within a given explanatory context there is clear delineation, then 
the distinction between mechanical part and resource is on as firm a footing as the concept of mechanical component more generally.

Sometimes the correct categorization becomes clear simply upon fixing the explanandum. Engine oil is a resource relative to the explanation of cooling: its thermal capacity varies with engine heat, and can be overwhelmed. RNA is a mechanism if you care about transcription, a cellular resource if you care about viral load. Both of those are explanations situated on roughly the same cellular-biological level, with interests separating out the correct characterization.

The spatial and temporal grain of the explanans also makes a difference (Bechtel and Mundale 1999). Jeeps are complex mechanisms to the motor pool, matériel to the general. A single CPU core is likely to be a mechanical part when it appears in explanations, because it is complex and distinctive. Aggregates of CPU cores, on the other hand, show up as resources when discussing distributed computing: then what is most relevant is sheer numbers of equipotent entities. Similarly so with background conditions. The mechanic can treat engine oil as a background condition on timescales of days and weeks. At longer timescales engine oil degrades from use, and so behaves more like a resource. The distinction between a short and a long timescale is a vague one, but not problematically so-once a day is definitely too frequently to change the oil, once a decade definitely too long. Similarly, differences in spatial scale might lead to differing categorizations: the rolling prairie is a resource to be divided for the farmer and a stable background condition for the ecologist. Again, concern with different spatiotemporal grain gives rise to different explanations.

The hierarchical decomposition inherent in mechanistic explanation can also treat a mechanistic part in one explanatory context as mechanism itself in another context. The capacities of the fuel rail can be explained by decomposing it into its mechanical parts. Sometimes resources can also be explained by decomposing them into mechanical parts: RAM is a resource for the programmer, but a collection of gates for the chip designer. The converse is also true. Water towers are (simple) mechanisms; part of the explanation of how they work treats the same object as including a very large spatial resource. And of course, as I have emphasized, most complex mechanisms will themselves decompose into a combination of resources and parts.

In sum, nothing counts as a mechanical part, resource, or background condition simpliciter. Instead, this is a delineation made within explanatory contexts, for specific purposes.

That said, however, I think the delineation of explanatory contexts themselves presents something of a challenge once we incorporate resources. I conclude with some reflections about the role of resources in explanations across contexts.

Recall that insofar as mechanists carve up the world, they work with local levels. As noted above, decomposition of a mechanism is meant to be made relative to a particular system behaving in certain ways. According to Craver, "levels of mechanisms are not monolithic divisions in the structure of the world" $(2007,190)$; hence "it makes no sense to ask if my heart is at a different level of mechanisms than my car's water pump because there is no mechanism containing the two" $(2007,191)$. Similarly, Bechtel notes that "[1]ocal identification of levels is sufficient for understanding levels in a mechanism and for capturing how mechanistic explanation is 
reductionist" $(2008,147){ }^{3}$ Local levels are meant to contrast with an older monolithic style of explanation (Oppenheim and Putnam 1958), on which there is a fixed stock of parts at each level of the world corresponding to each discipline.

Local decomposition arguably does fit better with how individual scientists investigate a particular phenomenon (Potochnik 2010). Yet the presence of resource explanations shows that local decomposition cannot be the whole story. For two distinct phenomena can interfere with one another just in case they each utilize the same limited resource. The irrigation system and the hydroelectric generator both draw from the same river; more cotton means more brownouts, and more power means less cotton. The mechanistic explanation of how the generator works includes a resource - the flow of water in the river-that is the same token resource that appears in mechanistic explanations of how the irrigation system works. Similarly so when taking dictation and reading aloud compete for the same orthographic representations (Botvinick and Cohen 2014).

There is an explanation about how the irrigation system works, and an explanation about how the dam works. I assume that the mechanist doesn't want to insist that these are insufficient unless we conjoin them into an unwieldy whole, which would thereby bring the river under the purview of a single mechanistic decomposition: that would undermine the point of local decomposition. But if we have two distinct explanations, then strictly local levels won't do. The appropriate constraints on a mechanistic decomposition are not just considerations like mutual manipulability. In many cases, we choose parts of a system because they are a resource that is part of some other system as well and resource competition is key to what we want to explain.

The same cross-explanation relevance is true of mechanical parts as well; resource competition just makes this especially vivid. Ultimately, as I emphasized in "Taxonomizing components" section, mechanistic explanation as a practice is valuable because it allows scientists to draw from a common store of components that appear in other explanations. Indeed (I suggest), this is precisely the value of mechanistic explanation (when it is available), rather than an unadorned causal/interventionist story. Resource explanations, while mechanistic, emphasize the importance of re-identifiable parts across distinct contexts.

Acknowledgements Thanks to Peter Clutton, Liz Irvine, Annelli Janssen, Meg Ivory, Chris Lean, Marco Viola, Elizabeth Schier, and participants at the 2014 Cognitive Science Kioloa workshop for helpful comments on earlier drafts. Thanks to Yellow Car, and indirectly to Karen Bennett, for inspiring many of the automotive examples. This research was supported by Australian Research Council Grant FT140100422.

\footnotetext{
3 See also Craver and Darden $(2013,88)$ and Bechtel and Richardson (2010, xix). A local sense of level arguably predates the mechanists. Marr's (1982) famous tripartite division of levels is drawn relative to a particular explanatory task; the lower levels are investigated in the context of sorting out the details of the computational level. Similarly, homuncular functionalism is concerned with functional decomposition relative to a starting capacity (Lycan 1990).
} 


\section{References}

Bechtel W (2008) Mental mechanisms: philosophical perspectives on cognitive neuroscience. Taylor \& Francis, New York

Bechtel W, Mundale J (1999) Multiple realizability revisited: linking cognitive and neural states. Philos Sci 66:175-207

Bechtel W, Richardson R (2010) Discovering complexity: decomposition and localization as strategies in scientific research. MIT Press, Cambridge

Botvinick MM, Cohen JD (2014) The computational and neural basis of cognitive control: charted territory and new frontiers. Cogn Sci 38(6):1249-1285

Campbell J (2007) An interventionist approach to causation in psychology. In: Gopnik A, Schulz L (eds) Causal learning: psychology, philosophy and computation. Oxford University Press, Oxford, pp 58-66

Campbell J (2010) II-control variables and mental causation. Proc Aristot Soc 110:15-30

Caramazza A (1986) On drawing inferences about the structure of normal cognitive systems from the analysis of patterns of impaired performance: the case for single-patient studies. Brain Cogn 5(1):41-66

Craver C (2007) Explaining the brain. Oxford University Press, New York

Craver CF, Darden L (2013) In search of mechanisms: discoveries across the life sciences. University of Chicago Press, Chicago

Cummins R (1975) Functional analysis. J Philos LXXI I(20):741-765

Cummins R (1983) The nature of psychological explanation. MIT Press, Cambridge

Duntemann J (2011) Assembly language step-by-step: programming with Linux. Wiley, New York

Haugeland J (1998) Mind embodied and embedded. Having thought: essays in the metaphysics of mind. Harvard University Press, Cambridge, pp 207-240

Klein C (2017) Brain regions as difference-makers. Philos Psychol 30(1-2):1-20

Lycan W (1990) The continuity of levels of nature. In: Mind and cognition: a reader. Basil Blackwell, Cambridge, pp 77-96

Marr D (1982) Vision: a computational investigation into the human representation and processing of visual information. WH Freeman, New York

Menzies P (2007) Causation in context. In: Corry R, Price H (eds) Causation, physics, and the constitution of reality: Russell's republic revisited. Oxford University Press, Oxford, pp 191-223

Messier C (2004) Glucose improvement of memory: a review. Eur J Pharmacol 490(1):33-57

Newman LA, Korol DL, Gold PE (2011) Lactate produced by glycogenolysis in astrocytes regulates memory processing. PloS ONE 6(12):1-11

Oppenheim P, Putnam H (1958) Unity of science as a working hypothesis. Minn Stud Philos Sci 2:3-36

Potochnik A (2010) Levels of explanation reconceived. Philos Sci 77(1):59-72

Shallice T (1988) From neuropsychology to mental structure. Cambridge University Press, Cambridge

Shapiro L (2005) The mind incarnate. MIT Press, Cambridge

Simon HA (1996) The sciences of the artificial, 3rd edn. MIT Press, Cambridge

Waters CK (2007) Causes that make a difference. J Philos 104(11):551-579

Woodward J (2010) Causation in biology: stability, specificity, and the choice of levels of explanation. Biol Philos 25(3):287-318 